This item was submitted to Loughborough's Research Repository by the author.

Items in Figshare are protected by copyright, with all rights reserved, unless otherwise indicated.

\title{
An efficient microwave-assisted chelation (MWAC) post-synthetic modification method to produce hierarchical $Y$ zeolites
}

\section{PLEASE CITE THE PUBLISHED VERSION}

https://doi.org/10.1016/j.micromeso.2020.110715

\section{PUBLISHER}

Elsevier BV

VERSION

AM (Accepted Manuscript)

\section{PUBLISHER STATEMENT}

This paper was accepted for publication in the journal Microporous and Mesoporous Materials and the definitive published version is available at https://doi.org/10.1016/j.micromeso.2020.110715.

\section{LICENCE}

CC BY-NC-ND 4.0

\section{REPOSITORY RECORD}

Abdulridha, Samer, Rongxin Zhang, Shaojun Xu, Aleksander Tedstone, Xiaoxia Ou, Jiacheng Gong, Boyang Mao, et al.. 2020. "An Efficient Microwave-assisted Chelation (MWAC) Post-synthetic Modification Method to Produce Hierarchical Y Zeolites”. Loughborough University. https://hdl.handle.net/2134/13623890.v1. 


\title{
An efficient microwave-assisted chelation (MWAC) post-synthetic modification method to produce hierarchical Y zeolites
}

\author{
Samer Abdulridha, ${ }^{\text {a† }}$ Rongxin Zhang, ${ }^{\text {a† }}$ Shaojun $\mathrm{Xu},{ }^{\mathrm{a}}$ Aleksander Tedstone, ${ }^{\mathrm{a}}$ Xiaoxia Ou, ${ }^{\mathrm{a}}$ Jiacheng Gong, ${ }^{\mathrm{a}}$ Boyang \\ Mao, ${ }^{\mathrm{b}}$ Mark Frogley, ${ }^{\mathrm{c}}$ Carlo Bawn, ${ }^{\mathrm{d}}$ Zhaoxia Zhou, ${ }^{\mathrm{e}}$ Xinran Zhang, ${ }^{\mathrm{d}}$ Sarayute Chansai, ${ }^{\mathrm{a}}$ Stuart M. Holmes, ${ }^{\mathrm{a}}$ Christopher \\ Hardacre, ${ }^{\text {a }}$ Arthur A. Garforth, ${ }^{\text {a Sihai Yang, }}{ }^{* d}$ Yilai Jiao, ${ }^{\text {aff }}$ Xiaolei Fan ${ }^{* a}$ \\ ${ }^{a}$ Department of Chemical Engineering and Analytical Science, School of Engineering, The University of Manchester \\ Oxford Road, Manchester, M13 9PL, United Kingdom \\ ${ }^{b}$ Department of Department of Engineering, University of Cambridge, JJ Thomson Avenue, Cambridge CB3 OFA, United \\ Kingdom \\ ${ }^{c}$ Diamond Light Source, Harwell Science and Innovation Campus, Didcot, Oxfordshire OX11 ODE, United Kingdom \\ ${ }^{d}$ Department of Chemistry, School of Natural Sciences, The University of Manchester, Oxford Road, Manchester, M13 \\ 9PL, United Kingdom \\ ${ }^{e}$ Loughborough Materials Characterisation Centre, Loughborough University, Loughborough, Leicestershire, LE11 \\ 3TU, United Kingdom \\ ${ }^{f}$ Shenyang National Laboratory for Materials Science, Institute of Metal Research, Chinese Academy of Sciences, 72 \\ Wenhua Road, Shenyang 110016, China Oxford Road, Manchester, M13 9PL, United Kingdom
}

\begin{abstract}
We report a low-cost, highly energy efficient microwave-assisted chelation (MWAC) method, which enabled the post-synthetic modification of synthetic zeolites for adopting hierarchical structures within minutes. Exemplified by Zeolite $\mathrm{Y}$, hierarchical $\mathrm{Y}$ zeolites prepared in this way showed exceptional specific external surface areas of $>300 \mathrm{~m}^{2} \mathrm{~g}^{-1}$ and mesopore volumes of $>0.46 \mathrm{~cm}^{3} \mathrm{~g}^{-1}$. Comparative assessments revealed that developed zeolites have shown significantly improved catalytic activities for catalysis involving large substrates, such as catalytic cracking and hydrocracking of plastics.
\end{abstract}

Keywords: hierarchical zeolites; post-synthetic treatment; microwave irradiation; catalysis

\section{Introduction}

Synthetic zeolites dominate the global zeolites market, and the worldwide synthetic zeolites market is estimated at several billions of U.S. dollars $[1,2]$. They are principally used in industries as heterogeneous catalysts [3]. For example, faujasite (FAU) type Y zeolites are the most important material in petroleum refinery catalysts for fluid catalytic cracking (FCC) and hydrocracking [4], being estimated to account for a share of $61.1 \%$ of the total market of synthetic zeolites in 2018 , regarding value [2]. The intrinsic micropores of these zeolites $(<1 \mathrm{~nm})$ lead to the long standing challenge of limitation on accessibility and diffusion during catalysis [5], for example, the reduced

\footnotetext{
* † These authors contributed equally. E-mails: sihai.yang@manchester.ac.uk (S.Y.); yljiao@imr.ac.cn (Y.J.); xiaolei.fan@manchester.ac.uk (X.F.)
} 
accessibility of active sites within the pore structure to the reactants and the slow diffusion rate of the products from the pores to the bulk media. The former leads to low reaction rates, while the latter yields coke that partially or completely blocks the active sites. Various strategies have been explored to enhance the accessibility of the active sites within zeolite frameworks [4, 6] such as: (i) design of zeolites with intrinsic large pores [7] or nanosized crystals [8, 9]; (ii) templating methods to endow zeolites with mesoporous features [10-12]; and (iii) post-synthetic treatments of pristine zeolites to create hierarchical meso-/micro-porous structures [13-15]. The last strategy based upon dealumination and desilication $[6,13,15-17]$ is robust and low-cost and thus particularly attractive for practical applications [16]. State-of-the-art post-synthetic dealumination and desilication are performed primarily by steaming, calcination and hydrothermal (HT) treatments in the presence of acids, bases or chelating agents (e.g., ethylenediaminetetraacetic acid, EDTA) [6, 16]. Although being effective, these processes are generally energy intensive and time-consuming [16], restricting the sustainable features of the resultant hierarchal zeolites. For creating mesopores in the aluminium-rich zeolites, especially zeolite Y (with the intrinsically low silicon-to-aluminum ratio, SAR, of $\sim 2.4$ ), compared with the steaming and acid leaching methods, chelation dealumination (also referred as chemical treatment) is relatively mild, being advantageous for making hierarchical $\mathrm{Y}$ with retained framework crystallinity and stability [14].

Microwave (MW) irradiation shows high efficiency in many chemical processes, such as the metal extraction from soils and plants [18, 19]. In zeolite chemistry, synthesis of zeolites under MW conditions has been proven to be more energy-efficient and time-saving than the conventional synthesis under the hydrostatic HT conditions [20]. Additionally, MW-assisted post-synthetic modification of zeolites and zeotypes (in the liquid-phase) regarding the acidity [21-24] and porosity [25-28] was also attempted using the mineral acids and bases. Especially, our recent work has shown that the combination of chelation dealumination with the microwave irradiation was highly efficient to prepare mesoporous zeolitic materials [29, 30]. 
Here we report the extended investigation of the post-synthetic treatment protocol involving chelation dealumination under MW irradiation (i.e., MW-Assisted Chelation, MWAC [29, 30]) for producing the modified synthetic zeolites with hierarchical pore structures. Specifically, and significantly, the MWAC method has enabled the preparation of hierarchical FAU zeolite Y on the minute timescale, and the obtained $\mathrm{Y}$ zeolites show exceptional hierarchical mesoporous features with the specific external surface area $\left(S_{\text {ext. }}\right)>300 \mathrm{~m}^{2} \mathrm{~g}^{-1}$ and mesopores volume $\left(V_{\text {meso }}\right)>0.46 \mathrm{~cm}^{3} \mathrm{~g}^{-1}$. As a result, the relevant zeolite catalysts exhibited notably enhanced catalytic activity and stability in catalytic cracking and hydrocatalytic decomposition (of plastics) reactions, compared with the commercial and conventional mesoporous $\mathrm{Y}$ zeolites. The MWAC method is generic for the post-synthetic modification of $\mathrm{Y}$ zeolite with different chelating agents, as well as other zeolites, showing significantly enhanced efficiency compared with the conventional HT processes.

\section{Materials and Methods}

Chemicals and materials used by this work are detailed in the Supporting Information, SI. The hierarchical Y zeolites were prepared using the MWAC method with EDTA as the model chelating agent in aqueous solutions, and followed by a workup procedure to remove the detrital materials from the framework. Specifically, MWAC treatment of zeolite $\mathrm{Y}$ was performed in a monomode microwave reactor (CEM Discover SP-D MW reactor) at $100{ }^{\circ} \mathrm{C}$ or $50{ }^{\circ} \mathrm{C}$ with the variable $\mathrm{MW}$ power input of maximum $150 \mathrm{~W}$. To prepare the mixture, $1.667 \mathrm{~g}$ zeolite $\mathrm{Y}$ was first charged into the EDTA aqueous solution $(25 \mathrm{ml}$, EDTA concentration $=0.05-0.2 \mathrm{M})$ in a $35 \mathrm{ml}$ Pyrex pressure vessel under stirring. The vessel containing the mixture was then inserted into the MW reactor and treated at the set temperature under stirring (at ca. $570 \mathrm{rpm}$ ) for various treatment times. After the MWAC treatment, the relevant zeolite samples were obtained after the workup procedure. Typically, the mixture was quenched using an ice water bath for $15 \mathrm{~min}$, and separated by centrifugation at 4,400 rpm to separate the solid from the solution. The resulting solid samples were washed using deionized water four times and dried at $70{ }^{\circ} \mathrm{C}$ in an oven overnight. The dry sample was then washed using 0.2 $\mathrm{M} \mathrm{NaOH}$ aqueous solution at $65^{\circ} \mathrm{C}$ for $30 \mathrm{~min}$ under stirring to remove the detrital material remained 
in the zeolite pores. Finally, the sample was washed thoroughly using deionized water to obtain the final products, which were dried at $70^{\circ} \mathrm{C}$ overnight prior to the characterisation. The resultant samples are denoted as MWAC-Y- $a-b-c$, in which $a, b$ and $c$ represent for the EDTA concentration (M), treatment time (min) and treatment temperature $\left({ }^{\circ} \mathrm{C}\right)$, respectively. By contrast, HT-Y-0.1-360-100 is the control mesoporous Y produced following the literature procedure $[13,14]$ by the conventional HT treatment with $0.1 \mathrm{M}$ EDTA at $100^{\circ} \mathrm{C}$ for $360 \mathrm{~min}(\mathrm{SI})$.

Materials prepared were comprehensively characterised using various techniques such as powder Xray diffraction $(\mathrm{XRD})$, nitrogen $\left(\mathrm{N}_{2}\right)$ physisorption, scanning electron and scanning transmission microscopy (SEM and S/TEM), Helium ion microscopy (HIM) and in situ infrared (IR) microspectroscopy, and relevant details of these techniques are provided in SI.

Comparative catalytic assessments of the zeolite catalysts were carried out using catalytic cracking of 1,3,5-triisopropylbenzene (TiPB, at $325^{\circ} \mathrm{C}$ under atmospheric pressure), hydro-catalytic cracking of polyproplylene and catalytic aldol condensation. All experimental information regarding catalysis is presented in SI in detail.

\section{Discussion}

By exposing zeolite $\mathrm{Y}$, suspended aqueous EDTA solutions, to $\mathrm{MW}$ radiation at $150 \mathrm{~W}$ and $100{ }^{\circ} \mathrm{C}$ for varying reaction time, hierarchical Y zeolites with mesoporous features were produced, surpassing those of both the parent and HT-Y-0.1-360-100 zeolites (Table 1). At $100{ }^{\circ} \mathrm{C}$, by varying the treatment time from 1 to 90 minutes, the obtained MWAC- zeolites show the specific external surface areas $\left(S_{\text {ext. }}\right)$ at $439 \pm 112 \mathrm{~m}^{2} \mathrm{~g}^{-1}$ and mesopores volumes $\left(V_{\text {meso }}\right)$ at $0.55 \pm 0.05 \mathrm{~cm}^{3} \mathrm{~g}^{-1}$, revealing an increase of a factor of $\sim 3$ in comparison to that of HT-Y-0.1-360-100. Indeed, these characteristic values of $S_{\text {ext. }}, V_{\text {meso }}$ and $V_{\text {total }}$ are amongst the highest ones reported to date for the post-synthetically modified Y zeolites (Table S1). By comparing the porosity of MWAC-Y zeolites, the effect of treatment time of the MWAC method is insignificant. For example, the value of $V_{\text {meso }}$ has increased by only $16 \%$ on extending the MWAC treatment time from 1 to 90 minutes. A control experiment using the MWAC 
method at $50{ }^{\circ} \mathrm{C}$ was performed; and the resulting MWAC-Y-0.1-1-50 showed distinct mesoporous features with $S_{\text {ext. }}=306 \mathrm{~m}^{2} \mathrm{~g}^{-1}$ and $V_{\text {meso }}=0.46 \mathrm{~cm}^{3} \mathrm{~g}^{-1}$ (Table 1), which are also higher than that of HT-Y. The findings demonstrate that the mesoporous characteristics of MWAC-Ys are not strictly related to the dielectric heating of the bulk water or the bulk thermal effect in the MWAC system. The micro- and meso-pore size distributions (PSDs) of MWAC-Ys can be readily tuned by varying the concentration of the chelating agent (Fig. S1, results related to the control blank experiments are shown in Figs. S2-S4 and Tables S1-S2), and the relevant crystallinity and solid yield data of the materials are presented in Fig. S4 and Table S2. Comparatively, samples obtained by the MWAC method using 0.1 M EDTA showed the optimal combination of micro- and meso-pores (Table 1), as well as the relative crystallinity of $62 \pm 1.2 \%$ and SAR of $3.9 \pm 0.9$ (Table S2).

Table 1. Textural properties of the parent zeolite $\mathrm{Y}$ and hierarchical $\mathrm{Y}$ zeolites.

\begin{tabular}{lllllll}
\hline \multirow{2}{*}{ Sample } & \multicolumn{2}{l}{ Specific surface areas $\left(\mathrm{m}^{2} \mathrm{~g}^{-1}\right)$} & \multicolumn{3}{l}{ Specific pore volumes $\left(\mathrm{cm}^{3} \mathrm{~g}^{-1}\right)$} \\
Parent Y & BET & $S_{\text {micro }}$ & $S_{\text {ext. }}$ & $V_{\text {total }}$ & $V_{\text {micro }}$ & $V_{\text {meso }}$ \\
HT-Y-0.1-360-100 & 867 & 858 & 9 & 0.36 & 0.35 & 0.01 \\
MWAC-Y-0.05-90-100 & 762 & 626 & 137 & 0.48 & 0.28 & 0.20 \\
MWAC-Y-0.1-90-100 & 930 & 804 & 312 & 0.73 & 0.33 & 0.40 \\
MWAC-Y-0.15-90-100 & 506 & 523 & 407 & 0.79 & 0.22 & 0.57 \\
MWAC-Y-0.1-30-100 & 986 & 415 & 571 & 0.82 & 0.24 & 0.58 \\
MWAC-Y-0.1-10-100 & 835 & 495 & 340 & 0.70 & 0.21 & 0.49 \\
MWAC-Y-0.1-1-100 & 822 & 491 & 331 & 0.70 & 0.21 & 0.49 \\
MWAC-Y-0.1-1-50 & 811 & 502 & 306 & 0.68 & 0.22 & 0.46 \\
\hline
\end{tabular}



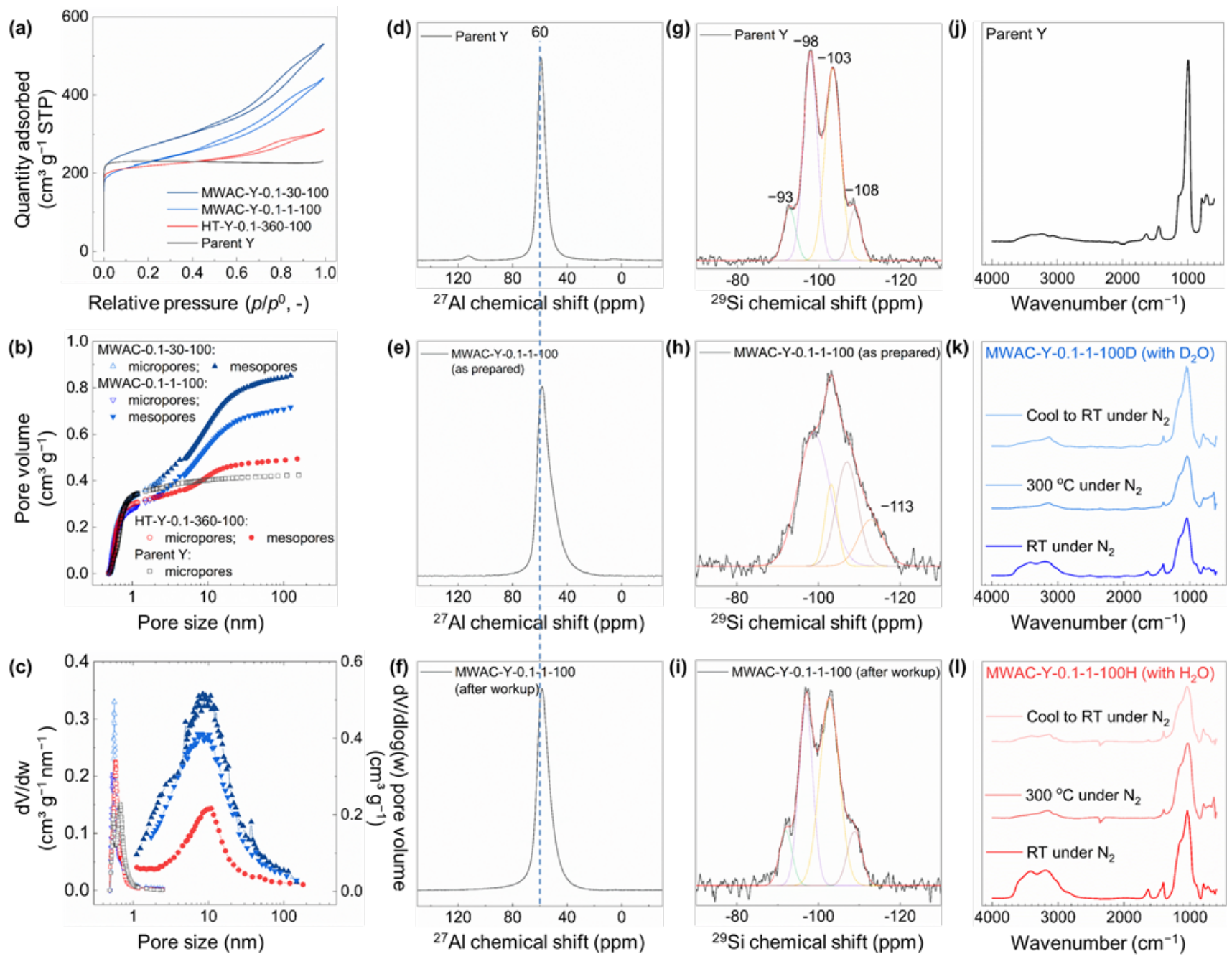

Fig. 1. Comparative characterisation of hierarchical $\mathrm{Y}$ zeolites under investigation. (a) $\mathrm{N}_{2}$ adsorption-desorption isotherms, (b) Cumulative PSDs, and (c) Differential PSDs of the parent Y, HT-Y and MWAC-Y zeolites; (d to f) $27 \mathrm{Al}$ MAS NMR spectra, and (g to i) 29Si MAS NMR spectra of the parent Y and MWAC-Y-0.1-1-100 zeolites; (j) Reference IR spectrum of the parent $\mathrm{Y}$ zeolite; ( $\mathrm{k}$ to $\mathrm{l}$ ) In situ IR micro-spectroscopic spectra of the as-synthesized MWAC-Ys prepared using hydrogen and deuterium labelling.

The presence of hierarchical mesopores of MWAC-Ys was evidenced by the observation of $\mathrm{H} 1$ type hysteresis loops in $\mathrm{N}_{2}$ adsorption-desorption isotherms of MWAC-Y-0.1-1-100 and MWAC-Y-0.130-100 compared with that of the parent Y and HT-Y (Fig. 1a), as well as the comparison of the cumulative pore volume (Fig. 1b) and differential PSD (Fig. 1c). The local Al environment of the zeolite across different stages of the treatment (after one minute) was studied by ${ }^{27}$ Al MAS NMR (Figs. 1d-1f), which suggests that, under the MW conditions, chelating agents can interact with the framework Al effectively (at ca. $60 \mathrm{ppm}$ ) to produce the tetrahedral non-framework Al sites [31], which was reflected by the broaden NMR spectrum (Fig. 1e). This is also evidenced by the ${ }^{29} \mathrm{Si}$ MAS NMR spectra of the parent and MW treated Y samples (Figs. 1g-1i), which show the disappearance 
of NMR signal at ca. -93 ppm for $\mathrm{Si}(3 \mathrm{Al})$ species and development of $\mathrm{Si}(4 \mathrm{Si})$ signal at ca. $-113 \mathrm{ppm}$ on the treatment. After the workup, MWAC-Y-0.1-1-100 showed similar ${ }^{27} \mathrm{Al}$ and ${ }^{29} \mathrm{Si}$ MAS NMR spectra (Figs. 1f and 1i) to that of parent Y (Figs. 1d and 1g), but signal broadening is observed for both spectra, suggesting the variation of local $\mathrm{Al} / \mathrm{Si}$ coordination within the framework after the posttreatment.

In situ infrared (IR) microspectroscopy study of MWAC-Y-0.1-1-100 zeolites treated using water and deuterium oxide $\left(\mathrm{D}_{2} \mathrm{O}\right)$ show that deuterium as the labelling isotope in the MWAC was not incorporated in the resulting framework, labelled as MWAC-Y-0.1-1-100D (Fig. 1k). Both MWACY-0.1-1-100H (MWAC-Y using water as the solvent, Fig. 1j) and MWAC-Y-0.1-1-100D show the main symmetric and asymmetric stretching (in the range of 3,000-3,600 $\mathrm{cm}^{-1}$ ) and bending (at ca. $1,635 \mathrm{~cm}^{-1}$ ) bands of lattice water, suggesting the MWAC method promotes the direct chelation of framework Al by EDTA which sequentially produced water [32]. These bands disappeared gradually by treating the samples under heating (up to $300{ }^{\circ} \mathrm{C}$ in a flow cell) under a dry $\mathrm{N}_{2}$ flow due to the desorption of the lattice water from the framework. In the -OH stretching region (Figs. 1k, 1j, S5 and S6), the band at $3,130 \mathrm{~cm}^{-1}$ emerged gradually during the in situ thermal treatment for both samples (in comparison to the spectrum of the parent zeolite, Fig. 1j), which confirmed the creation of the surface -OH groups through the MW-EDTA-zeolite interaction rather than the hydrolysis (Scheme 1). The $-\mathrm{OH}$ groups are stable at $300{ }^{\circ} \mathrm{C}$ and can be exchanged by deuterium (by introducing $\mathrm{D}_{2} \mathrm{O}$ vapour in $\mathrm{N}_{2}$ ), causing the predicted redshift of the IR spectra (Fig. S5). Importantly, by contrast, the post-synthetic treatment of zeolite Y using mineral acids (e.g. hydrochloric acid and nitric acid at 0.1 M) under MW irradiation was not effective in creating mesoporous structures (Table S1).

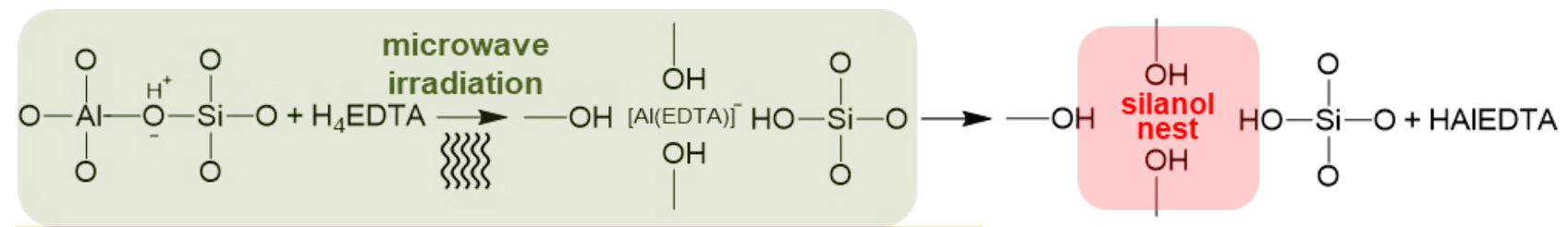

Scheme 1. Dealumination of Y zeolite by the MWAC method with EDTA. 
The hierarchical nature of MWAC-Y zeolites has also been studied by the scanning transmission electron microscopy (S/TEM) and helium (He) ion microscopy (HIM), as shown in Figs. 2, S7-S8. High-resolution TEM micrographs (Figs. 2a-2b) show distinct comparison between the parent zeolite and MWAC-Y-0.1-1-100. The parent Y shows the typical dense zeolite phase with well-aligned lattice fringes, while MWAC-Y demonstrates the co-existence of the intra-crystalline mesopores (ca. 5-10 nm) and crystal lattice fringes. The accessible hierarchical porosity of MWAC-Ys was confirmed by high-angle annular dark-field (HAADF) and focused He-ion beams imaging. Comparative TEM (Fig. 2c) and HAADF-S/TEM (Fig. 2d) images of MWAC-Y-0.1-30-100 show the opening of mesopores at the edges of the zeolite Y crystal. The feature was also evidenced by direct HIM imaging of the zeolite particle surface of MWAC-Y (Figs. 2e-2f), clearly showing the hierarchical mesopores that exist on the surfaces (similar features were also recorded by the low voltage scanning electron microscopy (SEM, Figs. S8e and S9). The crystalline nature of the MWAC$\mathrm{Y}$ is also confirmed by selected area electron diffraction (SAED) patterns of discrete spots (Fig. S8b).
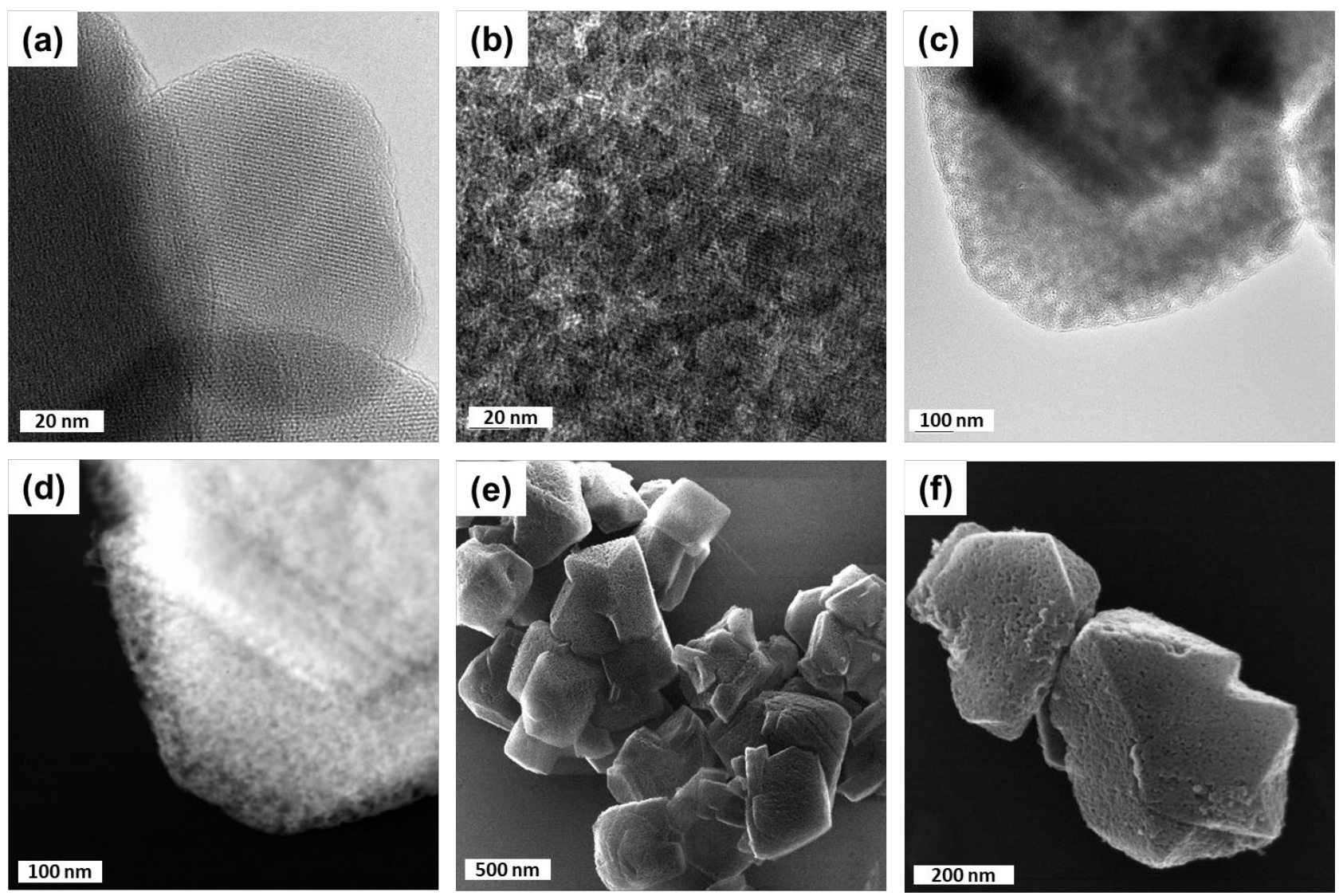

Fig. 2. (a) TEM micrograph of the parent $Y$; (b) TEM micrograph of the crystal domain and intracrystalline mesopores of MWAC-Y-0.1-1-100; (c and d) Comparative TEM and HAADF-STEM images of MWAC-Y-0.1-30-100; (e and f) HIM images of MWAC-Y-0.1-1-100. 
Stable hierarchical FAU Y zeolites (Figs. S10-S11) are important industrial catalysts, particularly for reactions involving bulky substrates. We assessed the catalytic properties of the selected MWAC-Ys using (i) catalytic cracking of a bulky aromatic hydrocarbon 1,3,5-triisopropylbenzene (TiPB), (ii) hydro-catalytic cracking of polypropylene (PP, using platinum, Pt, impregnated zeolites) and (iii) catalytic aldol condensation (for producing a bulky fragrance chemical, i.e. jasminaldehyde) (Figs. S12-S14) with direct comparison to the parent $\mathrm{Y}$ and/or commercial mesoporous $\mathrm{Y}$ and HT-Y zeolites. The MWAC-Y zeolites demonstrate exceptional performance on cracking TiPB (Fig. 3a), yielding quantitative conversions of TiPB as well as high selectivity to propylene (Fig. S13a), over the entire course of study. Conversely, the parent Y and HT-Y deactivated gradually due to the coke formation (Fig. S15). Given that the total acidity of the MWAC-Y zeolites is comparable to that of HT-Y (Fig. S11), the excellent catalytic activity of MWAC-Ys in cracking reactions is primarily due to the much enhanced hierarchical mesoporosity promoted by the MWAC method.
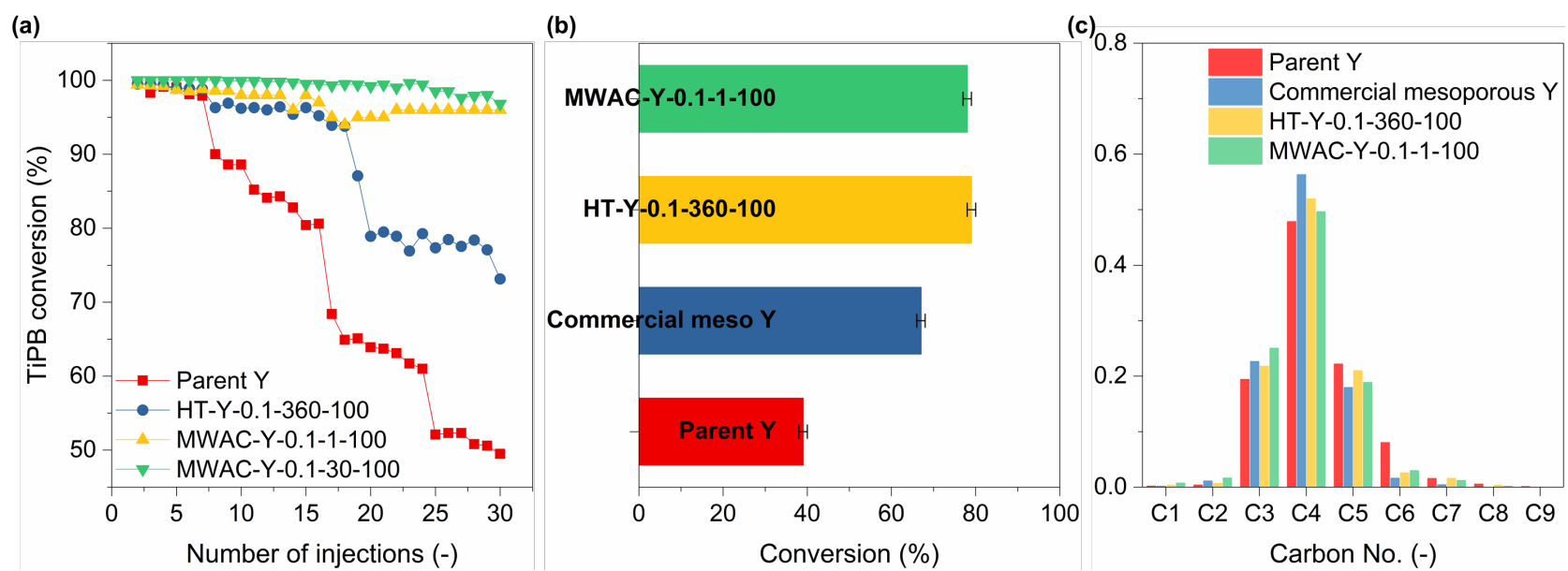

Fig. 3. Catalytic performance of the $\mathrm{Y}$ zeolite catalysts. (a) TiPB conversions over the $\mathrm{Y}$ zeolites as a function of injection numbers in catalytic cracking; (b) Polypropylene conversion over the Pt supported on Y zeolites catalysts in hydrocracking of polypropylene; (c) Weight average $\mathrm{Mw}$ of the pristine polypropylene and residual polymers from catalytic hydrocracking.

Comparative catalysis using the Pt supported on zeolite catalysts for the hydrocracking of plastics (polypropylene as the model plastic) was performed as well, as shown in Figs. 3b and 3c. The catalysts based on the MWAC-Y-0.1-1-100 and HT-Y-0.1-360-100 zeolites showed the comparable performance regarding the conversion of polypropylene (at $\sim 78 \%$ ), whereas the catalysts supported on the commercial mesoporous $\mathrm{Y}$ and Parent $\mathrm{Y}$ only enabled the conversions at about $67 \%$ and $39 \%$, 
respectively. All Pt catalysts supported on Y zeolites gave similar distributions of hydrocarbon products, centered at around C4, i.e. butane and isobutene, as shown in Fig. 3c. In addition to the light hydrocarbons produced (Fig. S14), gel permeation chromatography (GPC) analysis showed that the weight average molecular weight $\left(\mathrm{M}_{\mathrm{w}}\right)$ of the polymer retrieved after the hydrocracking reaction was reduced significantly compared with that of the pristine polypropylene. More importantly, the catalyst based on the MWAC-Y-0.1-1-100 showed the smallest $\mathrm{M}_{\mathrm{w}}$ value, being the most effective catalyst for depolymerisation.

\section{Conclusions}

The recently developed MWAC method is effective in modifying FAU Y for hierarchical mesoporosity with a wide range of chelating agents such as oxalic and citric acids (Table S4), and is particularly effective with multidentate clathro chelators, such as EDTA. Importantly, it is a generic method which is applicable to systems involving different types of zeolites, such as zeolite beta (with citric acid, Table S5). Although post-synthetic dealumination and desilication have been used as industrial methods for producing practical Y zeolites with the improved mesoporosity and stability, the process-efficiency of state-of-the-art methods has not yet been assessed comprehensively. The MWAC method is highly energy-efficient, exemplified by the preliminary comparison between the MWAC-Y-0.1-1-100 and HT-Y-0.1-360-100 zeolites. Specifically, regarding the production of $1 \mathrm{~g}$ hierarchical zeolite Y at the laboratory scale (SI), the MWAC method showed about 21-fold decrease in energy consumption (i.e. $\sim 0.02 \mathrm{kWh} \mathrm{g}^{-1}$ ) compared with that for the conventional hydrothermal method at $\sim 0.42 \mathrm{kWh} \mathrm{g}^{-1}$. Regarding the external surface area of hierarchical zeolite $\mathrm{Y}$, the MWAC method only requires $6.04 \times 10^{-5} \mathrm{kWh}$ to create $1 \mathrm{~m}^{2}$ external surface area, while the conventional method needs significantly more energy at $3.07 \times 10^{-3} \mathrm{kWh}$ (about 50 times as much as that by the MWAC). Therefore, the generic microwave promoted complexation effect highlights the possibility of developing transformative technologies for making hierarchical zeolites with great energy efficiency, as well as the industrial relevance. 


\section{Declaration of competing interest}

X.F. and Y.J. are inventors and S.A. and J.G are contributors on a patent application submitted by

The University of Manchester Intellectual Property Limited (UMIP) based on the intellectual property of the work (International Publication Number: WO 2020/053592 A1).

\section{CRediT authorship contribution statement}

X.F., Y.J. and S.Y.: Conceptualization, Methodology. S.A., R.Z., A.T., S.X., X.O., B.M., M.F., Z.Z., X.Z., S.C. and J.G.: Data curation. S.A., R.Z., A.T., and J.G.: Writing - original draft. X.F., S.Y., S.M.H., C.H., A.A.G., M.F. and Z.Z.: Writing - review \& editing. X.F.: Supervision.

\section{Acknowledgements}

S.A. was supported by The Higher Committee for Education Development in Iraq via a postgraduate research scholarship at The University of Manchester. Y.J. thanks the financial support from the China Scholarship Council (CSC) for his academic visiting fellowship in the UK (file no. 201604910181) and the National Key Research and Development Project (SQ2019YFE011366) for supporting his collaborative research.

\section{References}

[1] Flanagan, D. M. U.S. Geological Survey Minerals Yearbook 2018: Zeolites; February 2019.

[2] Global Synthetic Zeolites Market Forecast to 2023: Opportunities in Rising Demand for Adsorbents \& Recovering

Oil Prices 2018. https:/www.researchandmarkets.com/research/hjtmwf/global_synthetic?w=5 (accessed Mar 7, 2020).

[3] J. Weitkamp, Zeolites and catalysis, Solid State Ion. 131 (2000) 175-188.

[4] L. Kunhao, V. Julia, G.M. Javier, Realizing the Commercial Potential of Hierarchical Zeolites: New Opportunities in Catalytic Cracking, ChemCatChem 6 (2014) 46-66.

[5] P. Selvam, J. Sauer, B. Garrett, C. Campbell, R. van Santen, P. Davies, A.L. Miller, M. Bowker, G. Hutchings, D. Wotton, H.-J. Freund, M. Howard, M. Feaviour, R. Burch, A.M. Raspolli Galletti, E. Gross, F. Ivars Barcelo, A.

Kotarba, S. Kondrat, B. Weckhuysen, B. Majumdar, A. Bruix, N. Fischer, B. Gates, J. Moulijn, A. Roldan, N. Novak Tusar, T. Jakubek, D. Willock, M. Craven, K. Sethu, R. Catlow, R. Madix, H. Manyar, C. Friend, A. Corma, P. Wells, W. Ueda, A. Trunschke, R. Palmer, Designing new catalysts: synthesis of new active structures: general discussion, Faraday Discuss. 188 (2016) 131-159.

[6] D. Verboekend, N. Nuttens, R. Locus, J. Van Aelst, P. Verolme, J.C. Groen, J. Perez-Ramirez, B.F. Sels, Synthesis, characterisation, and catalytic evaluation of hierarchical faujasite zeolites: milestones, challenges, and future directions, Chem. Soc. Rev. 45 (2016) 3331-3352.

[7] A. Corma, M.J. Díaz-Cabañas, J. Martínez-Triguero, F. Rey, J. Rius, A large-cavity zeolite with wide pore windows and potential as an oil refining catalyst, Nature, 418 (2002) 514.

[8] A. Inayat, I. Knoke, E. Spiecker, W. Schwieger, Assemblies of Mesoporous FAU-Type Zeolite Nanosheets, Angew. Chem. Int. Ed. 51 (2012) 1962-1965. 
[9] H. Awala, J.-P. Gilson, R. Retoux, P. Boullay, J.-M. Goupil, V. Valtchev, S. Mintova, Template-free nanosized faujasite-type zeolites, Nat. Mater. 14 (2015) 447.

[10] J. Garcia-Martinez, M. Johnson, J. Valla, K. Li, J.Y. Ying, Mesostructured zeolite Y-high hydrothermal stability and superior FCC catalytic performance, Catal. Sci. Technol. 2 (2012) 987-994.

[11] I. Schmidt, A. Boisen, E. Gustavsson, K. Ståhl, S. Pehrson, S. Dahl, A. Carlsson, C.J.H. Jacobsen, Carbon Nanotube Templated Growth of Mesoporous Zeolite Single Crystals, Chem. Mater. 13 (2001) 4416-4418.

[12] S. Abdulridha, J. Jiang, S. Xu, Z. Zhou, H. Liang, B. Mao, Y. Zhou, A.A. Garforth, Y. Jiao, X. Fan, Cellulose nanocrystals (CNCs) as hard templates for preparing mesoporous zeolite $\mathrm{Y}$ assemblies with high catalytic activity, Green Chem. 22 (2020) 5115-5122.

[13] D. Verboekend, G. Vilé, J. Pérez-Ramírez, Hierarchical Y and USY Zeolites Designed by Post-Synthetic Strategies, Adv. Funct. Mater. 22 (2012) 916-928.

[14] D. Verboekend, T.C. Keller, S. Mitchell, J. Pérez-Ramírez, Hierarchical FAU- and LTA-Type Zeolites by PostSynthetic Design: A New Generation of Highly Efficient Base Catalysts, Adv. Funct. Mater. 23 (2013) $1923-1934$.

[15] Y. Jiao, L. Forster, S. Xu, H. Chen, J. Han, X. Liu, Y. Zhou, J. Liu, J. Zhang, J. Yu, C. D’Agostino, X. Fan, Creation of Al-Enriched Mesoporous ZSM-5 Nanoboxes with High Catalytic Activity: Converting Tetrahedral ExtraFramework Al into Framework Sites via Post Treatment, Angew. Chem. Int. Ed. (2020). DOI: 10.1002/anie.202002416. [16] R. Chal, C. Gérardin, M. Bulut, S. van Donk, Overview and Industrial Assessment of Synthesis Strategies towards Zeolites with Mesopores, ChemCatChem 3 (2011) 67-81.

[17] R. Zhang, P. Zhong, H. Arandiyan, Y. Guan, J. Liu, N. Wang, Y. Jiao, X. Fan, Using Ultrasound to Improve the Sequential Post-Synthesis Modification Method for Making Mesoporous Y Zeolites, Front. Chem. Sci. Eng. 14 (2020) 275-287.

[18] U. Chatreewongsin, Metal Extraction from Soil Samples by Chelation in a Microwave System. Ph.D. Thesis, Virginia Polytechnic Institute and State University, Blacksburg, Virginia, March 2000, pp. 137.

[19] S. Czarnecki, R.-A. Düring, Closed-vessel miniaturised microwave-assisted EDTA extraction to determine trace metals in plant materials, Int. J. Environ. Anal. Chem. 94 (2014) 801-811.

[20] X. Meng, F.-S. Xiao, Green Routes for Synthesis of Zeolites, Chem. Rev. 114 (2014) 1521-1543.

[21] M.D. González, Y. Cesteros, P. Salagre, F. Medina, J.E. Sueiras, Effect of microwaves in the dealumination of mordenite on its surface and acidic properties, Microporous Mesoporous Mater. 118 (2009) 341-347.

[22] M.D. González, Y. Cesteros, P. Salagre, Effect of microwaves on the surface and acidic properties of dealuminated zeolites, Phys. Procedia 8 (2010) 104-108.

[23] B.M. Chandra Shekara, B.S. Jai Prakash, Y.S. Bhat, Dealumination of Zeolite BEA under Microwave Irradiation, ACS Catal. 1 (2011) 193-199.

[24] M.D. González, Y. Cesteros, P. Salagre, Comparison of dealumination of zeolites beta, mordenite and ZSM-5 by treatment with acid under microwave irradiation, Microporous Mesoporous Mater. 144 (2011) 162-170.

[25] C.C. Pavel, W. Schmidt, Generation of hierarchical pore systems in the titanosilicate ETS-10 by hydrogen peroxide treatment under microwave irradiation, Chem. Commun. (2006) 882-884.

[26] S. Abello, J. Perez-Ramirez, Accelerated generation of intracrystalline mesoporosity in zeolites by microwavemediated desilication, Phys. Chem. Chem. Phys. 11 (2009) 2959-2963.

[27] V. Paixão, R. Monteiro, M. Andrade, A. Fernandes, J. Rocha, A.P. Carvalho, A. Martins, Desilication of MOR zeolite: Conventional versus microwave assisted heating, Appl. Catal. A 402 (2011) 59-68.

[28] Z. Hasan, J.W. Jun, C.-U. Kim, K.-E. Jeong, S.-Y. Jeong, S.H. Jhung, Desilication of ZSM-5 zeolites for mesoporosity development using microwave irradiation, Mater. Res. Bull. 61 (2015) 469-474.

[29] R. Zhang, D. Raja, Y. Zhang, Y. Yan, A.A. Garforth, Y. Jiao, X. Fan, Sequential Microwave-Assisted Dealumination and Hydrothermal Alkaline Treatments of Y Zeolite for Preparing Hierarchical Mesoporous Zeolite Catalysts, Top. Catal. 63 (2020) 340-350.

[30] S. Abdulridha, Y. Jiao, S. Xu, R. Zhang, A.A. Garforth, X. Fan, Mesoporous Zeolitic Materials (MZMs) Derived From Zeolite Y Using a Microwave Method for Catalysis, Front. Chem. 8 (2020) 482.

[31] Z. Yan, D. Ma, J. Zhuang, X. Liu, X. Liu, X. Han, X. Bao, F. Chang, L. Xu, Z. Liu, On the acid-dealumination of USY zeolite: a solid state NMR investigation, J. Mol. Catal. A 194 (2003) 153-167.

[32] G.T. Kerr, Chemistry of crystalline aluminosilicates. V. Preparation of aluminum-deficient faujasites, J. Phys.

Chem. 72 (1968) 2594-2596. 